Y. C. Minh and E. F. van Dishoeck, eds.

\title{
Optical and Near-Infrared Imaging of Jets and Outflows
}

\author{
Ji Yang and Yongqiang Yao \\ Purple Mountain Observatory, Nanjing 210008, PR China \\ National Astronomical Observatories, Chinese Academy of Sciences
}

\begin{abstract}
Jets and outflows are typical manifestations of the stellar mass loss process during the early stage of star formation. Optical and near-infrared (NIR) imaging of jets and outflows has become extremely popular recently, especially concerning large-scale surveys of Herbig-Haro (HH) objects, NIR imaging of jets and outflows driven by both low- and high-mass stars, and detailed imaging of jets and bow shocks at sub-arcsec resolution.

Optical and IR observations of jets and outflows have revealed (1) cavities evacuated by the outflow motion; (2) bow shocks inside and near the surface of the jets and outflows; (3) exciting sources; (4) circumstellar disks responsible for collimating the jets and outflows; (5) sometimes a cluster instead of a single source from which the jets and outflows initiate; (6) bursts of jets and outflows in some regions. Large-scale surveys of $\mathrm{HH}$ objects and outflows have been done in a number of nearby regions including Taurus, Orion, and Perseus. The prominent results of these studies range from the discovery of parsec-scale jets and outflows to their large-scale distribution. These studies also begin to reveal the relation between outflow activity and the large-scale distribution of young stellar objects.
\end{abstract}

\section{Introduction}

The ubiquitous existence of jets and outflows is demonstrated by the total number of jets and Herbig-Haro (HH) objects of up to 500 (Reipurth 1999). These objects provide a major step toward understanding one of the most important aspects of the mass loss process in the formation low-mass stars. A common feature of these jets and outflows, as observed in the optical and infrared, are the forbidden emission lines from atoms and ions as well as the vibration-rotation lines from molecules.

\section{Bow Shocks inside and near the Surfaces of Optical Jets and Out- flows}

A great variety of morphological structures have been found from these objects, including highly collimated jets, bow shocks, knots, and irregular shapes. Figure 1 demonstrates this with images of two HH objects, HH479 and HH480 (Yang et al. 1999). 

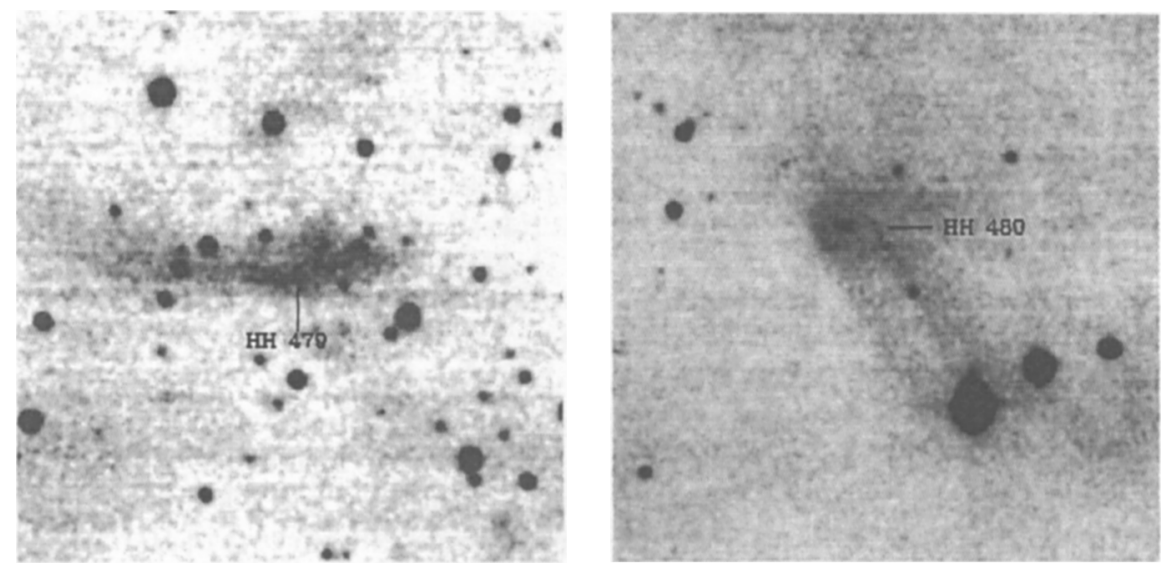

Figure 1. HH479 and HH480 in L1641 observed with a [SII] $\lambda \lambda 6717 / 6731$ filter. FOV is $7^{\prime}$, north is up and east is to the left. The images were taken with the $60 / 90$ Schmidt telescope at the $B e i$ jing Astronomical Observatory.

High-resolution observations revealed two main types of interaction: (1) Working surface between the ambient material and the high-velocity stellar wind, observed as the bow shock ahead of a jet along with a Mach disk upstream (e.g. the HST observation of HH47A, Heathcote et al. 1996). Shocks are resolved with a sharp $\mathrm{H} \alpha$ layer followed by a well separated [SII] post-shock cooling zone (Reipurth et al. 1997). (2) Internal shocks, generated within a high-velocity flow, resulting from the time variation of the mass-loss rate from a YSO. When the faster, newly ejected flow material overruns the previously ejected slowly-moving wind material, internal shocks will be excited.

Table 1. Properties of stellar optical jets.

\begin{aligned} & \hline Length $\leq 16 \mathrm{pc} \\ &$ Width $40 \mathrm{AU}\left(0 .{ }^{\prime \prime} 25\right.$ at $\left.140 \mathrm{AU}\right) \\ &$ Velocity $200-800 \mathrm{~km} \mathrm{~s}^{-1} \\ &$ Mach number $10-80 \\ &$ Electron density $400-2000 \mathrm{~cm}^{-3} \\ &$ Mass loss rate $10^{-8}-10^{-7} \mathrm{M}_{\odot} \mathrm{yr}^{-1} \\ &$ Dynamic age $2 \times 10^{2}-10^{5} \mathrm{yr} \\ &$ Luminosity of driving source $1-10^{5} \mathrm{~L}_{\odot} \\ &$\hline\end{aligned}

Internal shocks have been observed in HH111, HH34, and HH47 at highresolution (Heathcote et al 1996; Ray et al. 1996; Reipurth et al. 1997). Proper motion studies inferred a rather large shock velocity but the spectra do not show correspondingly high excitation, which can be explained by the internal flow in moving media. In HH111V, a flow of $400 \mathrm{~km} \mathrm{~s}^{-1}$ is overtaking a previous flow 


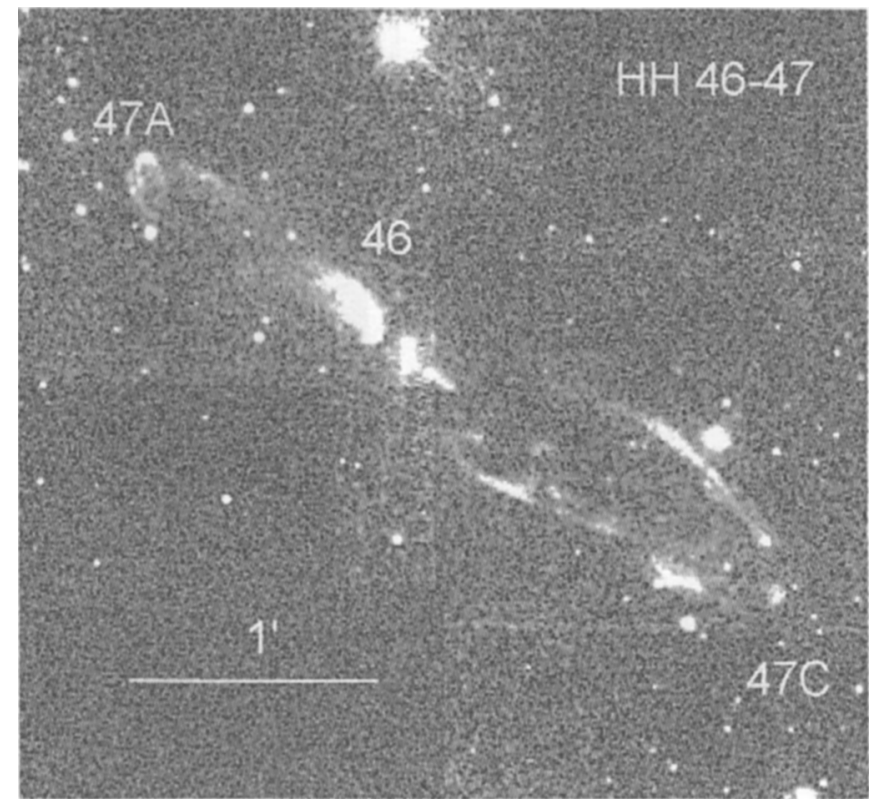

Figure 2. The $\mathrm{H}_{2} v=1-0 \mathrm{~S}(1)(2.12 \mu \mathrm{m})$ emission from the $\mathrm{HH} 46 / 47$ outflow. Note that the outflow cavity delineated by the shock is prominent in $\mathrm{HH} 47 \mathrm{C}$ (Chrysostomou et al. 1999).

speed of $300 \mathrm{~km} \mathrm{~s}^{-1}$ (Reipurth et al. 1997). Radial velocities, excitation degree, and ionization measurements proved that the bows and knotty structures inside a large-scale jet are due to the shocks moving inside previously-ejected tenuous wind material. The results support the validity of models which suggest that bow-shocks result from the dramatic time variation of probably both the velocity and the mass loss rate of high velocity winds over $10^{1-4} \mathrm{yrs}$. Alternative models, such as Kelvin-Helmholtz instabilities, cannot explain the details of the observed internal shock fronts.

Jets originate not only from low-mass stars, but also from intermediate to high-mass stars, e.g., Z CMa (Poetzel et al. 1989), HH80/81 (Marti et al. 1993), DR21 (Davis \& Smith 1996), and G192.16-3.83 (Devine et al. 1999). From the line width of $\mathrm{HH} 80 / 81$, it was found that the $\mathrm{HH}$ flow velocity may reach $700 \mathrm{~km} \mathrm{~s}^{-1}$ (HH80A; Heathcote et al. 1998). Table 1 summarizes the fundamental parameters of optical jets. Most of these parameters have been modified significantly during the last decade.

Beginning with HH34 (Bally \& Devine 1994), giant jets and HH objects have been discovered from large-scale surveys of star-forming regions. These systems extend over several parsecs. The total number of known systems has increased to more than 25 from the previous list of Bally \& Devine (1997). The largest one is perhaps from L1641-N, spanning $15.6 \mathrm{pc}$ (Reipurth et al. 1998). The inferred dynamical timescale of a giant HH jet system, $\tau=10^{4}-10^{5} \mathrm{yrs}$, is about one 

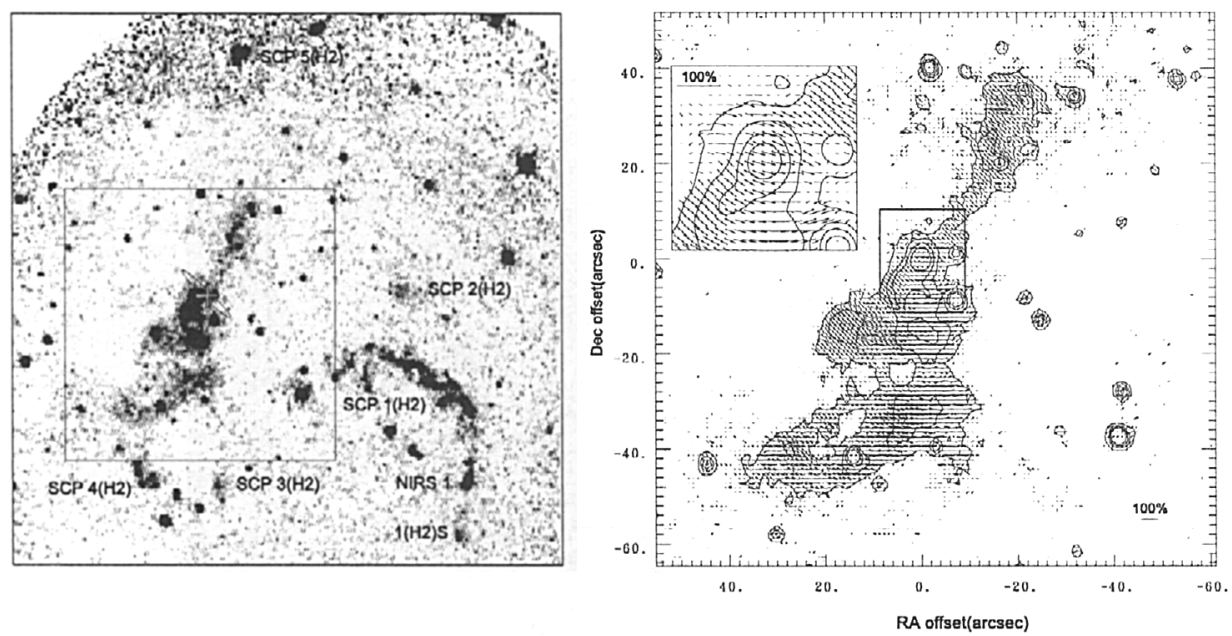

Figure 3. The $\mathrm{H}_{2} v=1-0 \mathrm{~S}(1)$ narrow band image of S187 IR in a field of $3.5^{\prime}$, and the $\mathrm{K}^{\prime}$ band polarization map for the bipolar nebula. The position of IRAS $01202+6133$ is marked by a plus and the $\mathrm{H}_{2}$ knots are labeled as SCP \#( $\left.\mathrm{H}_{2}\right)$ (Salas et al. 1998).

order of magnitude larger than that estimated from small-scalc jets, $\tau \sim 10^{3}$ yrs (Mundt et al. 1987). The observed scale raises the question of the mechanism to maintain the high degree of collimation over a much larger scale than previously treated. The distant impact of the giant $\mathrm{HH}$ jets may dramatically change our views on the dynamical and chemical influence of jet/outflow systems on the ambient medium, especially the giant molecular clouds.

\section{3. $\mathbf{H}_{2}$ Emission as an Alternative Probe of Jets and Outflows}

The $\mathrm{H}_{2} v=1-0 \mathrm{~S}(1)$ line emission is frequently observed towards jets and outflows from young stellar objects and is interpreted as a result of collisional excitation in shocked molecular gas at 2000-3000 K (e.g. Elias 1980; Schwartz et al. 1987). The coexistence of $\mathrm{H}_{2}$ shocked emission and $\mathrm{CO}$ outflows has been established to date from the narrow-band imaging survey of more than 6 dozen $\mathrm{CO}$ outflows (e.g. Davis et al. 1997 and references therein). Figure 2 shows the $\mathrm{H}_{2}$ emission from $\mathrm{HH} 46 / 47$. In $\mathrm{HH} 47 \mathrm{C}$, the $\mathrm{H}_{2}$ emission is more prominent than that observed in the optical.

Invisible, deeply embedded $\mathrm{H}_{2}$ jets can be imaged with currently available NIR arrays. It becomes clear that similar morphological structures as seen in the optical are also present in shock-excited NIR emission, particularly in molecular hydrogen lines. Good examples of such sources are L1448-mm (Davis et al. 1994), HH211 (McCaughrean et al. 1994), L1634 (Hodapp \& Ladd 1995), and IRAS05487+0255 (Garnavich et al. 1997). Shock-excited $\mathrm{H}_{2}$ emission in these objects is often observed coincident with, or close to, the regions of optical shocks and molecular outflows. 

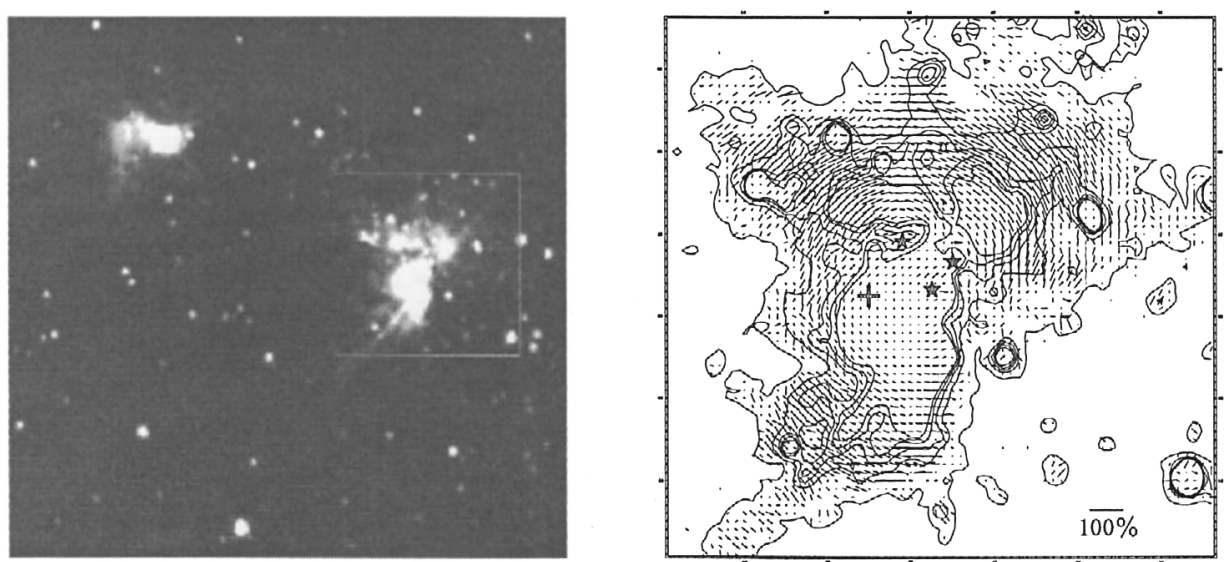

Figure 4. The $\mathrm{K}^{\prime}$ band image of AFGL6366S in a field of $3.4^{\prime}$, and the polarization map of the main nebula. A plus marks the position of IRAS $06056+2131$ and three stars mark the illuminating sources in the nebula. Note that the northern illuminating source remains deeply embedded and cannot be seen at wavelengths $\leq 2 \mu \mathrm{m}$ (Yao et al. 1999a).

\section{Nebulae and Cavities Associated with Jets and Outflows}

It has been well established that outflow sources are mostly associated with NIR nebulae (e.g. Hodapp 1994). The development of jets and collimated outflows will preferentially disrupt the parental cloud cores along the outflow axis and evacuate outflow cavities, through which the emission from central YSOs may be observed as bipolar reflection nebulae. Such reflection nebulae around low-mass stars are observed (e.g. Burrows et al. 1996; Lucas \& Roche 1997; Padgett et al. 1999). Even in a deeply embedded system, the cavity can be traced by NIR imaging. An example is the cavity following the blue-shifted outflow lobe in B335 (Hodapp 1998). In $\mathrm{HH} 47 \mathrm{C}, \mathrm{H}_{2}$ emission delineates a sharp cavity around the red-shifted lobe of the outflow, as shown in Figure 2.

The polarization patterns in the nebulae can identify loci of single and multiple scattering which are presumed to outline regions of lower and/or higher dust density. A recent example is S187, shown in Figure 3. The infrared nebula in S187 IR demonstrates a bipolar morphology, with jet and shell-like structures in the southern lobe (Jiang et al. 1999). Two $\mathrm{H}_{2}$ knots, SCP $4\left(\mathrm{H}_{2}\right)$ and $5\left(\mathrm{H}_{2}\right)$, are found to lie along the direction of the nebular elongation. Infrared emission from the nebula is highly polarized, with centrally symmetric vectors around a bright NIR point source. This NIR source coincides with the IRAS source in the region and can be identified as the exciting source of the outflow.

High resolution images in the near infrared also serve well to reveal complex structure of nebulae in heavily obscured and crowded cluster regions. Figure 4 presents an example of the AFGL6366S region (Yao et al. 1999a), associated with S247 in Gem OB1. The high resolution $\mathrm{K}^{\prime}$ band image reveals two nebulae associated with two CS cores (Carpenter et al. 1995), and a cluster point of sources apparently associated with the large western nebula. In the main nebula, 


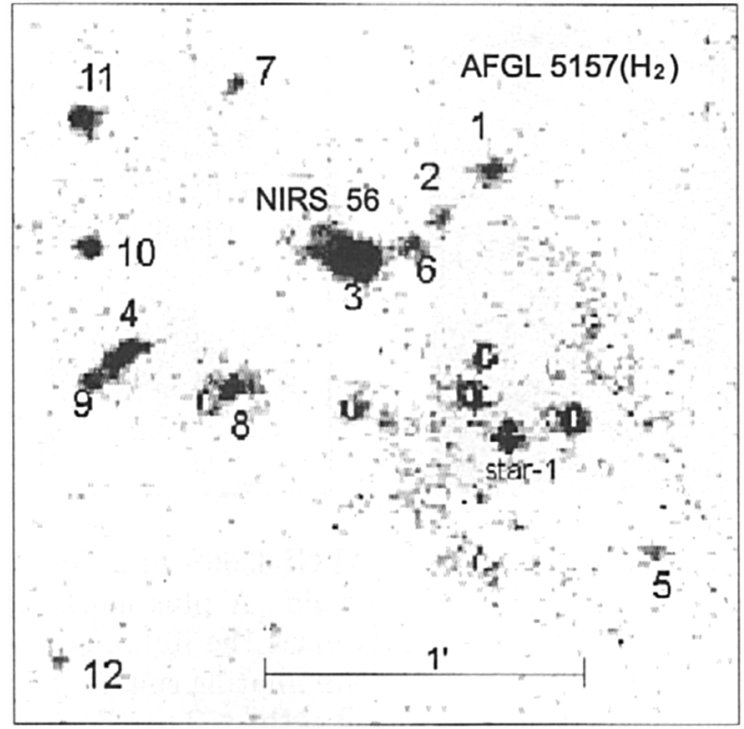

Figure 5. A group of $\mathrm{H}_{2}$-emitting jets and knots from the dense molecular core AFGL 5157 $\left(\mathrm{NH}_{3}\right)$ (from: Chen et al. 1999).

the polarization vectors delineate three nebular patches (in the south, NW and $\mathrm{NE}$ ), and reveal three illuminating sources responsible for each nebular patch. While the southern source is the brightest and associated with thermal (depolarized) emission, the NE source remains deeply embedded and cannot be seen at wavelengths $\leq 2 \mu \mathrm{m}$. The astrometry shows that the brightest source is located within the error ellipse of the IRAS source, and the embedded source coincides well with the $\mathrm{cm}$ continuum source and $\mathrm{H}_{2} \mathrm{O}$ maser in the region (Koempe et al. 1989).

Interestingly, the image resolves in the nebula many jet-like knots and streamers scattered mainly to the south and north, reminiscent of the exploding outflows in the BN-KL nebula (O'Dell et al. 1997). However, the polarimetric image shows that all the streamers are highly polarized, so that what is observed is primarily scattered light rather than line emission. These kind of streamers have been observed in other regions, such as NGC7538 (Yao \& Sato 1999), and could indicate the entrainment of cloud material by multiple outflows in high-mass and clustered star-forming regions.

\section{Bursts of Jets and Outflows in Star-Forming Regions}

Burst of HH objects have been revealed in NGC1333 (Hodapp \& Ladd 1995; Bally et al. 1996; Yan et al. 1998), NGC2068 (Zhao et al. 1999), and L1641 (Wang et al. 1999; Yang et al. 1999). In the active region of NGC1333, 30 


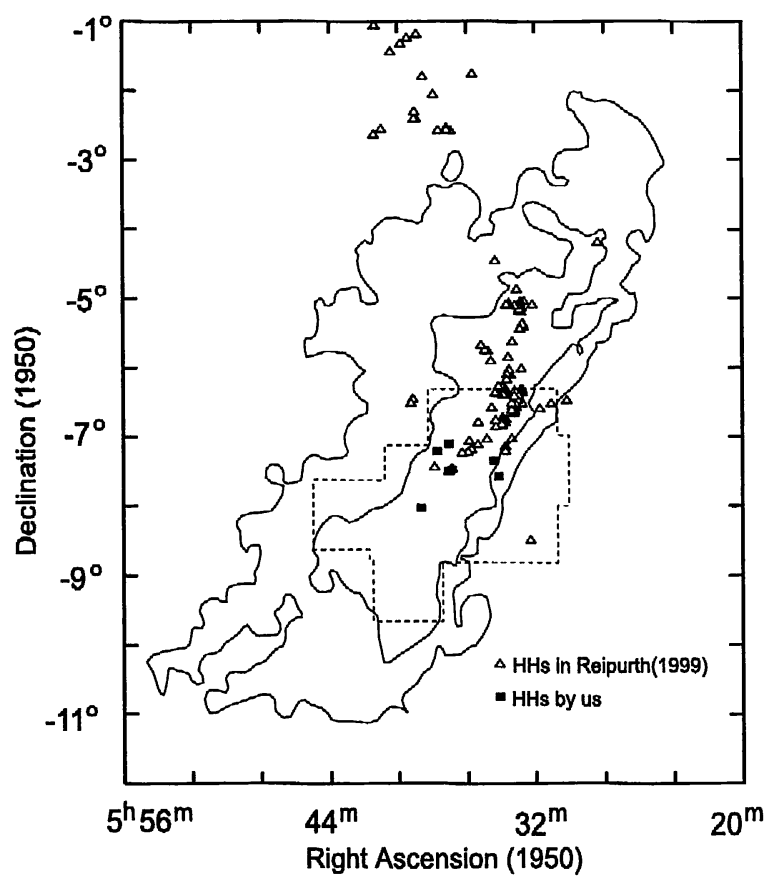

Figure 6. Large-scale distribution of optical jets and $\mathrm{HH}$ objects in L1641. Open triangles are sources listed in Reipurth (1999) and filled triangles are $\mathrm{HH}$ objects found from a survey by Wang et al. (1999) and Yang et al. (1999). Contour lines illustrate CO emission from the Columbia survey (Maddalena et al. 1986).

groups of HH objects were found (Bally et al. 1996; Yan et al. 1999). Similarly, in the prototypical case of the low-mass protocluster $\rho$ Oph, 12 jets/outflows have been discovered (Gomez et al. 1998). In L1641-N, Stanke et al. (1998) made an unbiased survey at $2.12 \mu \mathrm{m}$ and found a number of optically invisible $\mathrm{H}_{2}$ jets and knots, confirming that L1641-N is undergoing formation of a lowmass cluster (Strom et al. 1993).

In the dense molecular core AFGL 5157 $\left(\mathrm{NH}_{3}\right)$, Chen et al. (1999) found more than $11 \mathrm{H}_{2}$ jets and knots emanating from scattered locations and along different directions. The jet composed by $\mathrm{H}_{2}$ knots $1,2,6,3$ can be traced back to the very red infrared source NIRS 56 . However, there is apparently no single, prominent NIR source responsible for all the $\mathrm{H}_{2}$ emission. Therefore, this burst of $\mathrm{H}_{2}$ jets and knots could indicate that multiple star formation currently occurs in the dense molecular core AFGL 5157( $\left.\mathrm{NH}_{3}\right)$. Comparison with the J, H, \& $\mathrm{K}^{\prime}$ images shows that, in the same high-density clump, most of the potential exciting sources have not been found as point sources in the NIR and optical, indicative of a protocluster in nature. 
In L1211 (Yao et al. 1999b), a similar case was discovered for the multiple $\mathrm{H}_{2}$ jet. Most recently, mm continuum and line observations of L1211 dense core reveal a small cluster of at least four mm-sources associated with the $\mathrm{H}_{2}$ cmission and with resolved multiple molecular outflows (Tafalla et al. 1999). Therefore, in both cases of AFGL $5157\left(\mathrm{NH}_{3}\right)$ and $\mathrm{L} 1211$, it is very likely that we are viewing collapsing protoclusters just passing the infalling phase and exhibiting a burst of jet ejection.

Figure 5 illustrates the distribution of jets and HH objects over L1641. A prominent feature is the high concentration of jets and $\mathrm{HH}$ objects along a curved ridge extending from the southeast of NGC2023 to the middle east of L1641. We call this distribution enhancement the giant HH ridge. At present, it is not clear what kind of process determines such a large-scale distribution. A plausible mechanism is perhaps the compression by the large-scale shock from the OB association I Ori located in the northwestern part of the Orion molecular cloud (Blaauw 1964). If so, the shock compression could happen $\sim 4.6 \mathrm{Myrs}$ ago assuming a molecular shock propagating at $\sim 5 \mathrm{~km} \mathrm{~s}^{-1}$ from the approximatc location of the OB association. Such a timescale is close to the age of Orion OB associations inferred from the Hipparcos database, 2-11 Myrs (Brown et al. 1999).

Similar to the case of young stellar objects, the number of jets and $\mathrm{HH}$ objects can be used as a quantitative measure of the present-day stellar activity of star-forming regions. For example, Zhao et al. (1999) estimated that the surface number density of $\mathrm{HH}$ objects in the NGC2068/HH24 region reaches a very high value, $\sim 410 \mathrm{deg}^{-2}$. In contrast, the surface density is only $40 \mathrm{deg}^{-2}$ in NGC2071, about one order of magnitude lower. The results indicate the contrast of star formation rate at the current epoch is rather large over different regions.

Acknowledgments. The authors are grateful to Chris Davis for communication of their recent data and to Jiansheng Chen, Qin Zeng, Shuji Sato, Caipin Liu, and Licai Deng for various discussions. They acknowledge Hongchi Wang, Zhibo Jiang, Min Wang, and Bing Zhao for assistance and Ewine van Dishoeck for her kind encouragement.

\section{References}

Bally, J. \& Devine, D. 1994, ApJ, 428, L65

1997, in Herbig-Haro Flows and the Birth of Low-Mass Stars, IAU

Symp. 182, eds. B. Reipurth \& C. Bertout (Dordrecht: Kluwer), 29

Bally, J., Devine, D., \& Reipurth, B. 1996, ApJ, 473, L49

Blaauw, A. 1964, ARA\&A, 2, 213

Brown, A.G.A., Blaauw, A., Hoogerwerf, R., de Bruijne, J.H.J., \& de Zeeuw, P.T. 1999, preprint (astro-ph 9902234)

Burrows, C.J., Stapelfeldt, K.R., Watson, A.M., et al. 1996, ApJ, 473, 437

Carpenter, J.M., Snell, R.L., Schloerb, F.P. 1995, ApJ, 450, 201

Chen, Y., Yao, Y., Yang, J., et al. 1999, AJ, 117, 446

Chrysostomou, A. et al. 2000, MNRAS, in press

Davis, C.J., Dent, W.R.F., Matthews, H.E., Aspin, C.A., \& Lightfoot, J.F. 1994, MNRAS, 266, 933

Davis, C.J., Ray, T.P., Eislöffel, J., \& Corcoran, D. 1997, A\&A, 324, 263 
Davis, C.J. \& Smith, M.D. 1996, A\&A, 310, 961

Devine, D., Bally, J., Reipurth, B., Shepherd, D., \& Watson, A. 1999, AJ, 117, 2919

Elias, J.H. 1980, ApJ, 241, 728

Garnavich, P., Noriega-Crespo, A., Raga, A.C., \& Bohm, K. 1997, ApJ, 409, 752

Gomez, M., Whitney, B., \& Wood, K. 1998, AJ, 115, 2018

Heathcote, S., Morse, J.A., Hartigan, P., et al. 1996, AJ, 112, 1141

Heathcote, S., Reipurth, B., \& Raga, A.C. 1998, AJ, 116, 1940

Hodapp, K. 1994, ApJS, 94, 615 1998, ApJ, 500, L183

Hodapp, K. \& Ladd, E.F. 1995, ApJ, 453, 715

Jiang, Z., Yao, Y., Yang, J., et al. 1999, in preparation

Koempe, C., Baudry, A., Joncas, G., \& Wouterloot, J.G.A. 1989, A\&A, 221, 295

Lucas, P.W. \& Roche, P.E. 1997, MNRAS, 286, 895

Maddalena, R.J., Morris, M., Moscowitz, J., \& Thaddeus, P. 1986, ApJ, 303, 375

Marti, J., Rodriguez, L.F., \& Reipurth, B. 1993, ApJ, 416, 208

McCaughrean, M.J., Rayner, J.T., \& Zinnecker, H. 1994, ApJ, 436, L89

Mundt, R., Brugel, E.W., \& Bürke, T. 1987, ApJ, 319, 275

O'Dell, C.R., Hartigan, P., Lane, W.M., et al. 1997, AJ, 114, 730

Padgett, D.L., Brandner, W., Stapelfeldt, K.R., Strom, S.E., Terebey, S., \& Koerner, D. 1999, AJ, 117, 1490

Poetzel, R., Mundt, R., \& Solf, J. 1989, A\&A, 224, L13

Ray, T.P., Mundt, R., Dyson, J.E., Falle, S.A.E.G., \& Raga, A.C. 1996, ApJ, 468, L103

Reipurth, B. 1999, A general catalogue of HH objects, 2nd edition, http://casa.colorado.edu/hhcat

Reipurth B., Hartigan, P., Heathcote, S., Morse, J.A., \& Bally, J. 1997, AJ, 114, 757

Reipurth, B., Devine, D., \& Bally, J. 1998, AJ, 116, 1396

Salas, L., Cruz-Gonzalez, I., \& Porras, A. 1998, ApJ, 500, 853

Schwartz, R.D., Choen, M., \& Williams, P.M. 1987, ApJ, 322, 403

Stanke, T., McCaughrean, M.J., \& Zinnecker, H. 1998, A\&A, 332,307

Strom, K.M., Strom, S.E., \& Merrill, K.M. 1993, ApJ, 412, 233

Tafalla, M., Myers, P.C., Mardones, D., \& Bachiller, R. 1999, A\&A, in press

Wang, M., Zhao, B., Yang, J., Deng, L., \& Chen, J. 1999, Chinese Physics Lett., in press

Yan, J., Wang, H., Wang, M., Deng, L., Yang, J., \& Chen, J. 1998, AJ, 116, 2438

Yang, J., Wang, M., Deng, L., et al. 1999, in preparation

Yao, Y., Chen, Y., Yang, J., et al. 1999b, Acta Astron. Sinica, in press

Yao, Y. \& Sato, S. 1999, Acta Astron. Sinica, 40(1), 51

Yao, Y., Yang, J., Ishii, M., et al. 1999a, in preparation

Zhao, B., Wang, M., Yang, J., Wang, H., Deng, L., Yan, J., \& Chen, J. 1999, AJ, 118, 1347 


\section{Discussion}

D. Johnstone: Please comment on the correlation between the giant HH ridge in Orion and the dense molecular gas. Do you expect that these shocks are due to stars forming and producing jets or shocks directly related to the blast waves postulated to be running through the Orion region?

J. Yang: The giant HH ridge does not coincide well with the dense gas. A large fraction of the $\mathrm{HH}$ objects in the ridge can be traced back to some local young stellar objects. It indicates that the giant $\mathrm{HH}$ ridge in Orion is due to shocks from local stars instead of direct compression by a large-scale blast wave.

M. Burton: A comment on the lot of possible models for jets you presented. Fluorescence and shocks do exist together - in the source HH7 (Fernandes et al.) [a jet driven bow shock] and the bullets of Orion (Tedds et al.) [projectile driven bow shocks] a fluorescent signature is seen on top of the column density energy level signature for vibrational lines from $v \geq 3$. Presumably UV produced in a fast, dissociative jump-shock at the head of the bow fluorescently excited the hot, shocked $\mathrm{H}_{2}$ downstream from the head.

J. Yang: UV fluorescence is supposed to be present in the Cep A knots, HH7, HH47A, HH43, and even IRAS20126+4104. A more systematic NIR spectroscopy investigation may help to collect enough evidence. Sources with a high degree of excitation in the optical should be good candidates.

S. Pak: Is there any unified model to explain jets, outflows, and HH objects?

$J$. Yang: Not yet. A plausible framework of such a model may start from the jet-driven dynamics. 